\title{
Using social face-space to estimate social variables in real and artificial faces
}

\author{
Benjamin Balas \\ Psychology Department, North Dakota State University, Fargo, ND 58102, USA; Benjamin.balas@ndsu.edu \\ Josselyn Thrash \\ Psychology Department, North Dakota State University, Fargo, ND 58102, USA; josselyn.thrash@ndsu.edu
}

\begin{abstract}
Observers estimate a range of social characteristics from images of human faces. An important unifying framework for these judgments is the observation that a low-dimensional social facespace based on perceived valence and dominance captures most of the variance across a wide range of social evaluation judgments. However, it is not known whether or not this low-dimensional space can be used to infer the outcome of new social judgments. Further, the extent to which such social inference may differ across real and computer-generated faces is also unknown. We addess both of these issues by recovering valence/dominance axes from social judgments made to real and artificial faces, then attempt to use these coordinates to predict independent social judgment data obtained from new human observers. We find that above-chance performance can be achieved, though performance appears to be better with artificial faces than real ones.
\end{abstract}

Index Terms: Face recognition, Social evaluation, artificial faces.

\section{Introduction}

Observers make a wide range of social inferences from images of human faces. These include judgments of personality traits like aggression [1], competence [2] and trustworthiness $[3,4]$, as well as inferences regarding sexual orientation $[5,6]$, mental health $[7,8]$, and many other socially relevant aspects of behavior. These judgments vary a great deal in terms of their validity: Trustworthiness judgments, for example, do not tend to accurately predict individuals' tendency to behave in an honest fashion [9], while aggressive-looking faces do tend to engage in more realworld aggressive behaviors [10]. Despite this variability in the accuracy of social inferences based on facial appearance, observers appear to use such judgments to make important decisions. Election outcomes, for example, can be predicted using competence judgments made by naïve individuals who are unfamiliar with the faces in question [11-13]. Criminal sentencing outcomes also correlate with social judgments made solely from face images [14]. In a very real sense, first impressions thus matter a great deal: Observers make a wide range of social judgments given very little input [15] and then use those judgments to guide their behavior, their decision-making, and their opinions regarding unfamiliar individuals. Understanding the basis for these judgments is therefore an important means of understanding how visual perception and social interaction may mutually affect one another in real-world settings.
One important theoretical question regarding the large (and growing) list of social judgments observers make using face images is whether there exists a fundamental set of core social inferences that serve as the basis for others. That is, do observers maintain separate mechanisms for judging each of the various social properties described above, or is there a small set of core mechanisms that allow observers to estimate arbitrary social variables using these basic measurements? An influential theory advanced by Oosterhof and Todorov [16] suggests that two primary axes define social "face space": perceived trustworthiness (or valence) and dominance. This model of social face evaluation is based on the application of factor analysis to observers' ratings of 14 different social and personality characteristics from a set of face images, yielding these two axes which together account for a large fraction of the variance in the full data set. This main result has been replicated with multiple sets of face images, including ambient face images that incorporate substantial natural appearance variability [17] and both real and computer-generated faces images matched for identity [18]. In general, valence and dominance thus seem to be reasonable candidates for core mechanisms in social face space, and may serve as the basis for the full range of social evaluations that observers carry out. However, to our knowledge there has been little work examining whether the position of an individual face within this low-dimensional social face-space turns out to be a useful indicator of how that face will be evaluated with regard to other social attributes. Specifically, if you know the valence-dominance coordinates of a face, can you use that to predict how it will be rated or categorized on other social dimensions? While there have been several studies work examining the nature of the valence/dominance axes via correlation with other social attributes or other aspects of facial appearance [19], there has been little work using this model to predict the ratings independent observers assign to face regarding new traits.

Further, another issue regarding the development of the valence/dominance model is that there is variation across studies in terms of whether real or computer-generated faces were used to obtain a low-dimensional embedding of faces in social face-space. We suggest that this is an important consideration. While similar valence/dominance axes have been recovered from social evaluation judgments made to computer-generated [16] and real faces [17], there are important discrepancies between the evaluations made to real 
and artificial faces when face identity is matched. Trustworthiness judgments, for example, are disrupted by rendering faces with artificial appearance using morphable models [20] like the one implemented in the widely used FaceGen platform [21]. Considering valence/dominance judgments more broadly, Balas, Pacella \& Tupa [18] also reported that while both real and artificial faces yielded similar 2D models of social face judgments (with similar valence and dominance axes), the position of individual faces within that space varied substantially. That is, while we may evaluate faces according to their valence and dominance in each case, that evaluation plays out differently for individual faces depending on whether faces are rendered with photographic or computer-generated appearance. This has both practical consequences and theoretical importance. In terms of social interactions that may take place between a human observer and an artificial social agent, for example, understanding how social evaluation in general is based on basic estimates of personality characteristics is crucial to creating artificial agents that exhibit desirable social properties. Differences between how real and artificial faces are socially evaluated also highlight aspects of face recognition that depend on experience and tuning to realworld facial appearance. For example, social evaluation is known to depend on the race [14], sex [22], and attractiveness $[23,24]$ of face images, demonstrating that the way social inference proceeds from basic judgments of valence and dominance may differ as a function of perceptual or social experience. Artificial faces are an important way to examine these effects given that we can easily match the identity of face images across appearance conditions, thus controlling a number of socially-relevant appearance variables while manipulating the real/artificial appearane of the face.

Our goal in the current study was therefore to examine the extent to which valence/dominance information describing the position of identity-matched real and artificial faces in a social face-space could be used to predict other social judgments made by independent observers. Critically, we were interested in investigating both how well valence/dominance coordinates could be used to predict independent estimates of different social properties, and whether prediction accuracy varied as a function of real vs. artificial appearance. To address both of these questions, we asked two independent groups of observers to provide social evaluations of real and artificial face images: (1) One group provided the raw data for us to extract valence/dominance axes for real and artificial faces separately, and (2) A second group evaluated the same faces along several different social dimensions. Next, we used machine learning techniques to determine how well we could predict the evaluations made by the second group using the valence/dominance data provided by the ratings from the first group. Briefly, we find that generalizing from valence/dominance coordinates to other social judgments is challenging, and does vary as a function of real and artificial appearance.

\section{Methods \\ 2.1 Stimuli}

Our stimulus set was comprised of 128 images depicting real and computer-generated faces. Half of these images were created by applying a circular cropping window to full-color photographs of 32 men and 32 women. The original photographs were $2048 \times 1536$ pixels in size and depicted front-facing men's and women's faces that were free of make-up, facial piercings, or tattoos. Each photograph was also used to create a computer-generated face. We imported each individual's photograph into FaceGen, a widely-used commercial application based on a 3D morphable model of facial appearance [21] We identified fiducial points on each individual's face, yielding a 3D computer-generated version of each person. We applied a circular cropping window to each of these images, and resized all of our stimuli to a uniform size of $400 \times 400$ pixels (Figure 1).

\section{Original Face}

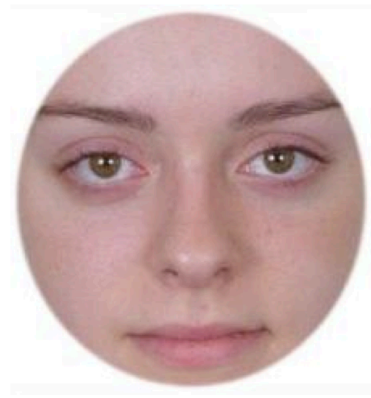

CG Face

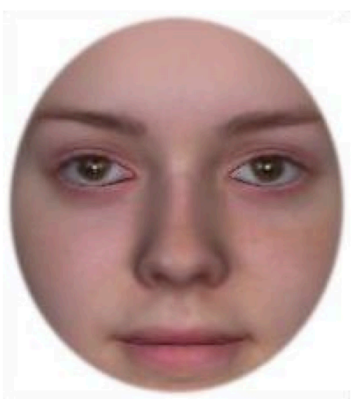

Fig. 1 An example of a real face (left) and an identity-matched artificial face (right) used in the current study.

\subsection{Participants}

In previous research using these stimuli [18], we recruited a sample of naive participants using the Amazon Mechanical Turk. The full description of this sample is provided in our prior report, but for our present purposes we note that we requested a total of 20 unique participants per image. While we did not request detailed demographic information from our respondents, typical MTurk samples tend to be more diverse than university samples and include roughly equal numbers of male and female participants.

We also recruited a new group of participants to provide novel social evaluation data that we required for the current study. These participants were recruited using the NDSU Undergraduate Psychology Study Pool, which is comprised of undergraduate students enrolled in sections of Introductory Psychology. We recruited a total of 50 participants (33 female), all of whom were between the ages of 18-25 years and self-reported normal or corrected-to-normal vision. Written informed consent was obtained from all participants, and participants received course credit for volunteering for this study. 


\subsection{Procedure}

Regarding our first sample of participants, our testing procedures are described in detail in Balas, Pacella \& Tupa [18]. Briefly, participants recruited via the Amazon Mechanical Turk were asked to rate individual faces on a 1-7 Likert scale according to 14 different personality traits. These traits corresponded to the social judgment used in Todorov \& Oosterhof [16] to obtain valence-dominance axes using dimensionality-reduction techniques. The full list of traits is included below (Table 1).

\begin{tabular}{l}
\hline Personality Traits \\
\hline Aggressive \\
Weird \\
Emotionally Stable \\
Intelligent \\
Caring \\
Responsible \\
Trustworthy \\
Confident \\
Dominant \\
Attractive \\
Unhappy \\
Sociable \\
Mean \\
-
\end{tabular}

Table 1 The personality traits we asked participants to assess in Balas, Pacella \& Tupa (2017) to obtain valence/dominance axes for real and artificial faces.

We tested our second sample of participants in person in the Psychology Department at NDSU. In this task, participants were asked to complete five blocks of a new social evaluation judgment using either the real or artificial faces described above. During each block of the experimental session, we asked participants to provide a binary judgment (yes/no) regarding whether each person was likely to have high/good values for a specific social variable. Specifically, we asked participants to evaluate: (1) The physical health of each face, (2) The mental health of each face, (3) Competence, (4) Trustworthiness, (5) Aggression. Within each block, individual faces were presented one at a time, and remained onscreen until the participant made a judgment via pre-determined keys on the keyboard. Participants were seated in front of a 1200x800 LCD display at a viewing distance of approximately $50 \mathrm{~cm}$, at which distance the stimuli subtended approximately 5 degrees of visual angle. All stimuli were presented on a uniform white background, and block order was rotated across participants so that each social variable appeared equally often in each position within the full experiment. Each face image was presented once per block in a pseudorandomized order, and participants either completed the task using real images or artificial images (no participant saw both real and artificial faces). All stimulus display and response collection procedures were implemented using the Psychtoolbox v3.0 for MATLAB [2527].

\section{Results}

3.1 Obtaining valence-dominance coordinates via dimensionality reduction.

As reported in Balas, Pacella \& Tupa [18], we used the rating data provided by our participants on AMT to obtain valencedominance axes for real and artificial faces separately. To summarize the main outcomes of this analysis, we applied principal components analysis to the 14-dimensional data obtained by calculating the average rating per attribute per picture for each image in the two stimulus sets. In each case, we found that the first two principal components captured a substantial portion of the total variance (approximately 50\% for both real and artificial faces). Furthermore, the coefficients comprising each of the two axes in each case supported an interpretation of these dimensions in terms of valence and dominance, similar to prior work using both artificial faces [16] and real faces [17] For our current purposes, we used this low-dimensional embedding of the real and artificial stimuli to assign valence-dominance coordinates so that each image was described in terms of its projection along these two axes. These coordinates served as the basis for our subsequent attempts to use machine learning techniques to predict the judgments made by the independent observers who rated faces according to the novel attributes described above.

\subsection{Assigning attribute labels for new social judgments.}

To examine how well valence-dominance coordinates could be used to predict social judgments made by independent observers, we used the data provided by our second sample of participants to split each set of faces into partitions reflecting "high" and "low" values for each attribute. Specifically, for each of the five social attributes we asked this sample of participants to judge, we calculated the proportion of participants that labeled each image as having a high/good value for that attribute. This yielded a value bounded by $0-1$ per image, per attribute. Next, we carried out a median split of these values such that the images with proportions in the upper half of the distribution were assigned a value of +1 and the remaining images were assigned a value of -1 . We thus obtained 5 different partitions of the same images for both real and artificial faces, reflecting which faces received the most endorsements for each attribute.

To compare item-level ratings across real and artificial face appearance, we calculated pairwise correlations for each attribute using the proportions calculated by the procedure described above (Figure 2). We observed non-significant correlations between real and artificial ratings for most attributes: Physical health $-\mathrm{R}=-0.019, \mathrm{p}=0.88$; Mental health $=\mathrm{R}=-0.15, \mathrm{p}=0.23$; Competence - $\mathrm{R}=-0.004, \mathrm{p}=0.97$; Aggression - $\mathrm{R}=0.092, \mathrm{p}=0.47$. We did, however, observe a significant correlation between real and artificial trustworthiness evaluations $(\mathrm{R}=0.45, \mathrm{p}=0.002)$, which is consistent with prior results demonstrating that while trustworthiness judgments are disrupted by artificial 
appearance [20], there remains a relationship between real and artificial trustworthiness judgments.
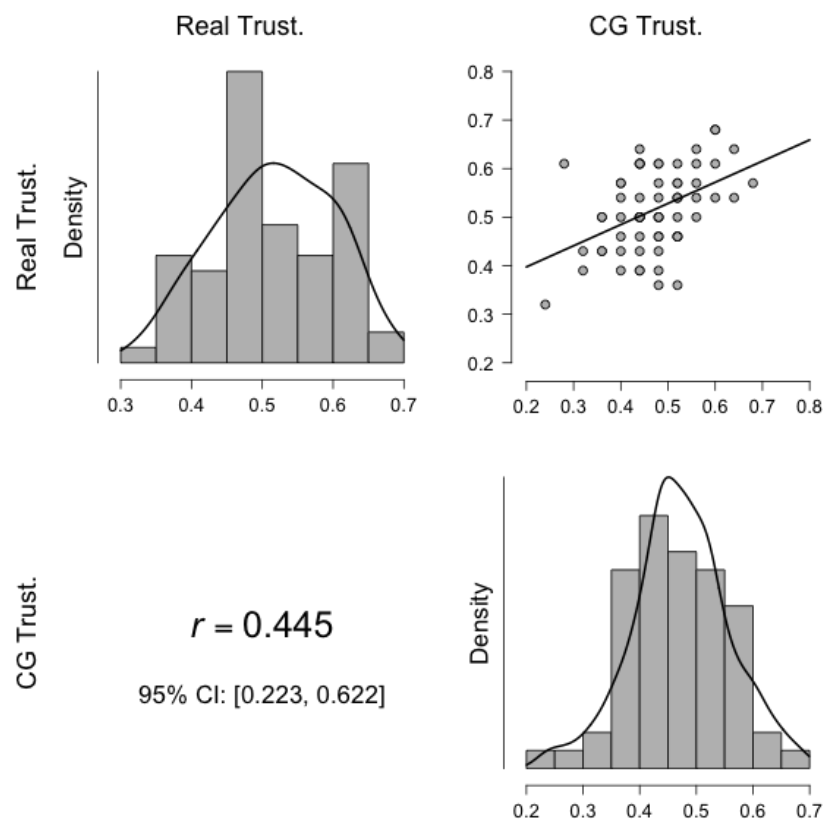

Fig. 2 Plots of marginal densities for the proportion of high "Trustworthiness" assignments made to individual real (upper left) and artificial faces (lower right) in our main task. The positive correlation between the proportion of high "Trustworthiness" assignments for real and artificial faces (upper right) reached significance (lower left, Pearson's R and 95\% confidence interval).

It is important to note that the lack of correlations between real and artificial face evaluations in this context does not imply anything about the possibility for using valence/dominance coordinates to extrapolate to new social judgments. These first analyses instead allow us to examine the relationship between social evaluations made to real faces and artificial faces. The lack of an obvious relationship between real and artificial social judgments does not preclude the existence of sufficiently coherent structure within each category to support accurate classification of high/low attribute assignments on the basis of valence/dominance coordinates in each case. What these results do indicate, however, is that social evaluation is broadly disrupted by artificial appearance: Identity-matched real and artificial faces tend to receive highly variable values across our set of social judgments.

\subsection{Classifying social attributes using valence/dominance coordinates.}

Our primary goal in the current study was to investigate the predictive value of valence/dominance coordinates for determining whether real and artificial faces would be evaluated as being "high" or "low" with regard to new social attributes. To do so, we trained separate linear SVMs for each attribute, in each case evaluating our classification accuracy using the labels obtained for each item based on the median split described above and the valence/dominance coordinates for each face. Specifically, we used 10-fold cross-validation to randomly partition the data into nonoverlapping subsets such that each SVM was trained on $90 \%$ of the data, with the remaining $10 \%$ held in reserve as a test set. The subset held out as test data was rotated across the full set of partitions so that accuracy was computed based on correct category labels for the entire data set. Because the partitioning procedure was stochastic, we carried out 100 iterations of this procedure for each combination of appearance category (real or artificial) and attribute (Physical health, mental health, competence, trustworthiness, and aggression). Finally, we calculated bootstrap estimates of the mean and standard deviation of each distribution of accuracy values. These values, broken down by appearance category and attribute, are displayed in Figure 3.

We can see from the figure that the most prominent features of these results is the overall lack of above-chance performance. For the most part, classification accuracy of these traits hovers quite close to $50 \%$ for both real and artificial faces. The categorization of both trustworthiness and competence reaches above-chance levels, however, though in the case of trustworthiness, this appears to be more so the case with regard to real faces.

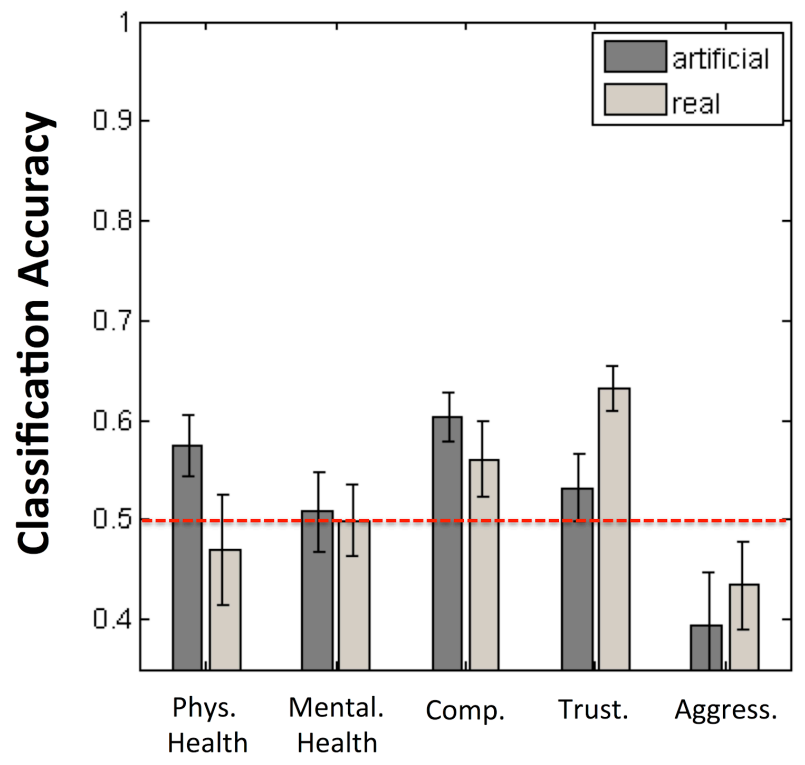

Fig. 3 Classification accuracy for each of the five independently assessed personality traits using valence/dominance coordinates to represent each item. Error bars represent bootstrap estimates of the standard deviation.

As an alternative to our use of a linear SVM, we also attempted to carry out the same pattern classification task using a simple nearest-neighbor classifier. One advantage of using nearest-neighbor classification is that compared to discriminant methods that evaluate the separability of classes, nearest-neighbor classification makes fewer assumptions about the underlying distributions of data points across categories. We thus also carried out the crossvalidation procedures describe above using a k-nearest neighbor classifier with values of $\mathrm{k}$ between 1 and 5 . These 
outcomes were qualitatively similar, so in Table 2 we present the results we obtained with $\mathrm{k}=5$, which yielded the best categorization performance overall.

\begin{tabular}{lll}
\hline Trait & Real Faces & CG Faces \\
\hline Physical Health & $0.53(0.028)$ & $0.60(0.027)$ \\
Mental Health & $0.45(0.032)$ & $0.59(0.026)$ \\
Competence & $0.52(0.027)$ & $0.58(0.025)$ \\
Trustworthiness & $0.56(0.023)$ & $0.64(0.026)$ \\
Aggression & $0.62(0.022)$ & $0.66(0.027)$ \\
\hline
\end{tabular}

Table 2 Bootstrapped means and standard deviations (in parentheses) for nearest-neighbor classification of personality traits based on valence/dominance coordinates for real and artificial (CG) faces.

Briefly, it is evident from this table that categorization using this approach tended to be more accurate than the results obtained using a linear SVM. In contrast to the results we obtained with a linear SVM, this outcome provides more support for the use of a valence/dominance space as the basis for further social evaluation of real and artificial faces.

There are of course many other kinds of classifiers that could be applied to the dataset described here, and also many different choices of parameter values than we have explored. In the interests of making it possible for others to pursue different classification strategies than we have reported here, our full dataset is available online via the Open Science Framework (link: https://osf.io/uh6x5/). This includes all of the real and artificial faces used in the current tasks, the average trait ratings per face on each of the 14 dimensions used in [16] and [18], the valence/dominance coordinates we recovered from our data, and the categorization proportions we used to assign labels for our pattern classification analysis. We have also included our analysis code to support independent replication of our approach.

\section{DISCUSSION}

Our results demonstrate a number of interesting properties regarding how social evaluation is carried out in identitymatched real and artificial faces. First, we observed that there was generally no relationship between the extent to which a real face would tend to receive "High" ratings for social attributes and the extent to which it's computer-generated partner would receive the same ratings. For physical health, mental health, competence, and aggression, these correlations were non-significant. Only trustworthiness evaluations were correlated across real and artificial face appearance in this task, which is consistent with prior results [18].

Why should there be a relationship between real and artificial face trustworthiness, but not these other attributes? One possibility is that the visual features used as proxies for trustworthiness judgments are largely preserved when real faces are rendered with artificial appearance, leading to similar assessments of trustworthiness based on this information. With respect to trustworthiness in particular, perceived emotional valence in neutral expression images is known to be a robust proxy variable for social evaluation [28,29]: Faces that look a bit happy when making a neutral expression tend to be rated as more trustworthy than faces that look a bit sad or angry when they are making a neutral expression. Is emotion categorization well-preserved following the application of computer-generated face appearance? Indeed, most emotions are categorized accurately in artificial faces [30], which may help explain why trustworthiness judgments that may be based in part on these aspects of appearance are also reasonably consistent across our appearance categories. We note, however that judgments of aggression based on facial appearance depend critically on the width-to-height ratio of the face [10], defined as the interpupillary distance divided by the height between the eyes and mouth. This simple geometric ratio is also matched closely across real and artificial faces, yet we see no evidence of a correlation between aggression evaluations across the two face types. Given the close matching of shape properties across our identity-matched real and artificial faces, it may be the case that some aspects of skin pigmentation and related surface properties are disrupted when faces are rendered with artificial appearance, and these must carry important information for social evaluation. Skin smoothness, for example, influences observers' evaluation of some social variables [31], and computer-generated faces frequently appear to have smoother skin than real face images. In general, these basic results comparing the social evaluation of real faces and artificial faces suggest a number of interesting avenues to explore, particularly with regard to understanding the visual information that leads artificial faces to receive different social evaluations than real ones.

Our second main result is that our attempts to categorize individual faces as being "High" or "Low" in each of the 5 attributes we evaluated independently largely ended in failure when we used a linear SVM, but were more successful when we applied k-nearest neighbors classification. This result suggests that high and low trait datapoints were not generally linearly separable in valence/dominance space, but were sufficiently "clumpy" within local neighborhoods to support limited generalization. This result provides tentative support for the hypothesis that this particular social face-space serves as the basis for social evaluation considered more broadly. Classification accuracy was still quite low across all of the judgments we included in our main task, but we argue that this is consistent with many of the results described in the social evaluation literature with human subjects. In many of these studies, the main results indicate that participants perform significantly above-chance, but with small effect sizes. For example, with regard to mental health outcomes, Kleiman \& Rule [8], reported mean A' values of $\sim 0.53$ (significantly greater than a chance value of 0.5 ) in a $2 \mathrm{AFC}$ task in which participants were asked to determine whether or not face images depicted individuals who had committed suicide or not. In other tasks examining observers' abilities to predict sexual orientation [32], extroversion [33], and other social variables, performance is similar: Participants tend to significantly exceed chance performance as a group, 
but the absolute level of performance is not very high. We suggest, therefore, that the results we report here are not so dissimilar to human performance and provide some support for the utility of a valence/dominance space for extrapolation to other social judgments from face images.

Besides observing above-chance classification results for many of the judgments that we considered here, we also observed a fairly consistent difference between performance with real faces and performance with artificial faces. Specifically, when we used k-nearest neighbor classification to measure performance, artificial faces led to higher accuracy than real faces across all tasks. One interpretation of this outcome is that human observers use information beyond valence/dominance estimates to assign new social labels to real faces, but tend to rely more heavily on these values for artificial faces. This could be due to the lack of some visual information in artificial faces (detailed surface texture, for example), making it more difficult for observers to use additional mechanisms for social evaluation in computer-generated images. Alternatively, this could also be a by-product of limited exposure to artificial faces: Limited tuning to artificial face appearance may compromise observers' ability to process these faces efficiently, leading to increased reliance on basic aspects of facial structure that are reflected in valence/dominance judgments. One interesting way to disentangle these alternatives would be to explore how performance in this task is affected by imposing varying levels of blur on real face images. If artificial face judgments are predicted more accurately because the rendering process leaves out critical information that supports more complex judgments, removing some of that information by degrading real faces should lead to a similar outcome. To our knowledge, there has been little work examining how social evaluation from face images is affected by manipulations of image quality. While limited presentation time appears to have a minimal effect on social evaluations $[3,12,15,33]$ changing image quality may have a larger impact on performance.

Regardless of the mechanisms supporting this difference in performance across real and artificial faces, our results have implications for the way artificial agents may be perceived by human observers. Specifically, our results suggest that social evaluation of artificial faces is substantially simpler than the evaluation of real faces: Valence/dominance information carries a good bit of information about other social variables. While the lack of linear separability that we observed makes it more challenging to use these variables to make strong predictions regarding how other judgments will unfold, the higher performance we observed using k-NN classification suggests that observers are using a reduced set of more basic measurements to make social inferences from artificial faces. This may make it easier for designers to focus their efforts on these aspects of social face evaluation when designing artificial agents, as these fundamental measurements play a larger role in how computer-generated faces are evaluated more broadly.

\section{CONCLUSION}

Our results demonstrate that using valence/dominance facespace as the basis for inference regarding other social face judgments is possible. The position of faces within this $2 \mathrm{D}$ space is of some use for predicting how those faces will be evaluated along other social dimensions. However, these coordinates supported better classification performance for artificial faces, suggesting that the evaluation of real faces may depend on a broader set of mechanisms. Overall, these results are consistent with an emerging picture of artificial faces as an example of an out-group relative to real face images, but also suggest some practical advantages to working with artificial faces in settings where social judgments are important.

\section{ACKNOWLEDGEMENTS}

Special thanks to Ganesh Padmanabhan and Dan Gu for technical assistance. Thanks also to Amanda Auen for comments regarding the study design and consultation regarding recruitment.

\section{REFERENCES}

1. J.M. Carre, C.M. McCormick \& C.J. Mondloch, Facial Structure Is a Reliable Cue of Aggressive Behavior, Psychological Science. 20, 11941198 (2009)

2. A.B. Sussman, K. Petkova, \& A. Todorov, Competence ratings in US predict presidential election outcomes in Bulgaria, Journal of Experimental Social Psychology. 49, 771-775 (2013)

3. A. Todorov, M. Pakrashi, \& N.N. Oosterhof, Evaluating faces on trustworthiness after minimal time exposure, Social Cognition. 227, 813-833 (2009)

4. M. Dzhelyova, D.I. Perrett, \& I. Jentzsch, Temporal dynamics of trustworthiness perception, Brain Research. 1435, 81-90 (2012)

5. N.O. Rule \& N. Ambady, Brief exposures: Male sexual orientation is accurately perceived at $50 \mathrm{~ms}$, Journal of Experimental Social Psychology. 44, 1100-1105 (2008)

6. K.O. Tskhay \& N.O. Rule, Accurate identification of a preference for insertive versus receptive intercourse from static facial cues of gay men, Archives of Sexual Behavior. 42, 1217-1222 (2013)

7. K.A. Fowler, S.O. Lilienfeld \& C.J. Patrick, Detecting psychopathy from thin slices of behavior, Psychological Assessment. 21, 68-78 (2009)

8. S. Kleiman \& N.O. Rule, Detecting Suicidality from Facial Appearance, Social Psychological and Personality Science. 4, 453-460 (2012)

9. N.O. Rule, A.C. Krendl, Z. Ivcevic \& N. Ambady, Accuracy and Consensus in Judgments of Trustworthiness: Behavioral and Neural Correlates, Journal of Personality and Social Psychology. 104, 409-426 (2013)

10. J.M. Carre \& C.M. McCormick, In your face: Facial metrics predict aggressive behaviour in the laboratory and in varsity and professional hockey players, Proceedings of the Royal Society B: Biological Sciences. 275, 2651-2656 (2008)

11. C.C. Ballew \& A. Todorov, Predicting political elections from rapid and unreflective face judgments, PNAS. 104, 17948-17953 (2007)

12. C.Y. Olivola \& A. Todorov, Elected in 100 milliseconds: Appearancebased trait inferences and voting, Journal of Nonverbal Behavior. 34, 83-110 (2010)

13. A.C. Little, R.P. Burriss, B.C. Jones \& S.C. Roberts, Facial appearance affects voting decisions, Evolution and Human Behavior. 28, 18-27 (2007)

14. J.L. Eberhardt, P.G. Davies, V.J. Purdie-Vaughns \& S.L. Johnson, Looking deathworthy: Perceived stereotypicality of Black defendants predicts capital-sentencing outcomes, Psychological Science. 17, 383386 (2006) 
15. J. Willis \& A. Todorov, First impressions: Making up your mind after 100 ms exposure to a face, Psychological Science. 17, 592-598 (2006)

16. N.N. Oosterhof \& A. Todorov, The functional basis of face evaluation, Proceedings of the National Academy of Sciences of the USA. 105, 11087-11092 (2008)

17. C.A.M. Sutherland, J.A. Oldmeadow, I.M. Santos, J. Towler, D.M. Burt \& A.W. Young, Social inferences from faces: Ambient images generate a three-dimensional model, Cognition. 127, 105-118 (2013)

18. B. Balas, L. Tupa \& J. Pacella, Measuring social variables in real and artificial faces, Computers in Human Behavior. 88, 236-243 (2018)

19. A. Todorov, R. Dotsch, J.M. Porter, N.N. Oosterhof \& V.B. Falvello, Validation of Data-Driven Computational Models of Social Perception of Faces, Emotion. 13, 724-738 (2013)

20. B. Balas \& J. Pacella, Trustworthiness perception is disrupted in artificial faces, Computers in Human Behavior. 77, 240-248 (2017)

21. V. Blanz \& T. Vetter, Face recognition based on fitting a $3 D$ morphable model, IEEE Transactions on Pattern Analysis and Machine Intelligence. 25, 1063-1074 (2003)

22. K.R. Macapagal, H.A. Rupp \& J.R. Heiman, Influences of observer sex, facial masculinity, and gender role identification on first impressions of men's faces, Journal of Social and Cultural Evolutionary Psychology. 5, 92-105 (2011)

23. S.K. Johnson, K.E. Podratz, R.L. Dipboye, Gibbons, E. Physical attractiveness biases in ratings of employment suitability: Tracking down the "beauty is beastly" effect, The Journal of Social Psychology. 150, 301-318 (2010)

24. A.C. Little, S.C. Roberts, B.C. Jones \& L.M. Debruine, The perception of attractiveness and trustworthiness in male faces affects hypothetical voting decisions in wartime and peacetime scenarios, Quarterly Journal of Experimental Psychology. 65, 2018-2032 (2012)

25. D.H. Brainard, The Psychophysics Toolbox, Spatial Vision. 10, 433-436 (1997)

26. D.G. Pelli, The VideoToolbox software for visual psychophysics: Transforming numbers into movies, Spatial Vision. 10, 437-442 (1997)

27. M. Kleiner, D. Brainard, D. Pelli, A. Ingling, R. Murray \& C. Broussard, What's new in Psychtoolbox-3, Perception. 36, 1 (2007)

28. N.N. Oosterhof \& A. Todorov, Shared perceptual basis of emotional expressions and trustworthiness impressions from faces, Emotion. 9, 128-133 (2009)

29. C. Said, N. Sebe \& A. Todorov, Structural resemblance to emotional expressions predicts evaluation of emotionally neutral faces, Emotion. 9, 260-264 (2009)

30. M. Dyck, M. Winbeck, S. Leiberg, Y. Chen, R.C. Gur, K. Mathiak, Recognition Profile of Emotions in Natural and Virtual Faces, PLoS ONE. 3(11), e3628 (2008)

31. E. Tsankova \& A. Kappas, Facial Skin Smoothness as an Indicator of Perceived Trustworthiness and Related Traits, Perception. 45, 400-408 (2016)

32. N.O. Rule, N. Ambady \& K.C. Hallett, Female sexual orientation is perceived rapidly, accurately, and automatically from the face and its features, Journal of Experimental Social Psychology. 45, 1245-1251 (2009)

33. P. Borkenau, S. Brecke, C. Mottig \& M. Paelecke, Extraversion is accurately perceived after a 50-ms exposure to a face, Journal of Research in Personality. 43, 703-706 (2009) 